\title{
3D X-ray Microscopy of Nuclear Energy Materials
}

\author{
Nikolaus Cordes, Joshua Kane and Aaron Craft
}

Idaho National Laboratory, Idaho Falls, Idaho, United States

Three dimensional (3D) X-ray imaging, also referred to as X-ray computed tomography (CT), is a datarich characterization technique that can provide surface and subsurface spatial information in a nondestructive manner. ${ }^{1-3}$ Simply put, absorption-contrast radiographs of a sample are collected as a function of either sample rotation or source-detector rotation (i.e., gantry systems). The radiographs are mathematically reconstructed to provide a 3D dataset (i.e., tomogram) which can be used for sample/part inspection of defects, image quantification, or as inputs into finite element analyses. The spatial resolution range for this technique is wide and can vary based on the choice of the X-ray source (e.g., micro-focus or nano-focus tube sources, synchrotron sources, linear accelerators, etc.), magnification modality (e.g., geometric or optical), and detection method (e.g., flat panel detector, objective lens coupled to a chargecoupled device (CCD) camera). Sub-micrometer resolution can be achieved with 3D X-ray microscopy, which typically employs a micro-focus X-ray source and objective lens/CCD camera system to achieve finer resolutions than what is achievable with most traditional X-ray tomographic imaging systems. ${ }^{4}$

3D X-ray imaging of nuclear energy materials, especially fuel, is a difficult endeavor. Mass attenuation coefficients of high-Z materials, such as uranium, are problematically large relative to what is readily accessible with most commercially available, laboratory-based micro-focus X-ray sources, thus limiting the practical maximum X-ray path length to a few millimeters at most. While this physical limitation impedes the nondestructive microstructural imaging of fuel rods, sub-micrometer scale 3D X-ray microscopy can be employed to characterize nuclear fuel samples of the appropriate size. In addition, surrogate samples can be used to accelerate imaging, for example, in order to investigate analogous fuel processing methods. ${ }^{5}$

Idaho National Laboratory (INL) has recently acquired a ZEISS Xradia 520 Versa X-ray microscope capable of nondestructively imaging a wide range of materials over four orders of magnitude in length scales $\left(10^{1}-10^{-2} \mathrm{~cm}\right)$ in 3D. This presentation will give an overview of the application of this laboratorybased imaging technique to nuclear energy materials, such as uranium-zirconium alloys, zirconium systems, Si-C fiber composites, mock fuel systems, and Advanced Gas Reactor fuel systems (e.g., tristructural isotopic (TRISO) particles and compacts).

\section{References}

1. Maire, E.; Withers, P. J., Quantitative X-ray tomography. International Materials Reviews 2014, $59(1), 1-43$.

2. Stock, S. R., Recent advances in X-ray microtomography applied to materials. International Materials Reviews 2008, 53 (3), 129-181.

3. Dunsmuir, J. H.; Bennett, S.; Fareria, L.; Mingino, A.; Sansone, M., X-ray microtomographic imaging and analysis for basic research. Powder Diffraction 2006, 21 (2), 125-131.

4. Merkle, A. P.; Gelb, J., The Ascent of 3D X-ray Microscopy in the Laboratory. Microscopy Today 2013, 21 (2), 10-15.

5. $\quad$ Lowe, T.; Bradley, R. S.; Yue, S.; Barii, K.; Gelb, J.; Rohbeck, N.; Turner, J.; Withers, P. J., Microstructural analysis of TRISO particles using multi-scale X-ray computed tomography. Journal of Nuclear Materials 2015, 461, 29-36. 